\title{
Körperschallsensorik für die echtzeitfähige Erkennung von strukturschädigenden Aufbruchversuchen in Automaten
} Johann Harmstorf ${ }^{1}$, Lars Kleditsch ${ }^{1}$, Jakob Happel ${ }^{1}$, Alexander Raaz ${ }^{1}$, Karl-Ludwig Krieger ${ }^{1}$

${ }^{1}$ Universität Bremen, Institut für Theoretische Elektrotechnik und Mikroelektronik, Otto-Hahn-Allee 1, 28359 Bremen, Deutschland

\section{Zusammenfassung}

Gegenstand dieser Veröffentlichung ist die Entwicklung eines echtzeitfähigen körperschallbasierten Sensorsystems. Hier werden die analoge und digitale Signalverarbeitung, der Analog-Wake-Up, die Spannungsversorgung sowie die Einbindung der externen Schnittstellen beschrieben. Zudem werden die speziellen Eigenschaften der verwendeten PVDF-Folien im Kontext des Einsatzbereichs gezeigt. Dieser Sensor soll zur automatischen Erkennung, Klassifizierung und Bewertung von strukturschädigenden Angriffen auf Automaten verwendet werden. Dabei wurde insbesondere darauf hingearbeitet, die strukturschädigenden Aufbruchversuche der Automaten in Echtzeit zu erkennen, um die Täter frühzeitig von der weiteren Zerstörung des Automaten abzuhalten. Über die Sensoren werden die Körperschallsignale, die bei unterschiedlichen Angriffen auf die Automaten entstehen, aufgenommen und anschließend unter Berücksichtigung des aktuellen Automatenzustandes bewertet. Da die Körperschallsignale je nach Art des Angriffs unterschiedlich sind, soll so nicht nur der Vorgang eines Angriffs erkannt werden, sondern auch die Art des Angriffs unterschieden und dann zielgerichtete Gegenmaßnahmen eingeleitet werden. Auf diese Weise soll ein effektiverer Schutz vor der vollständigen Zerstörung des Automaten gewährleistet werden, als dies mit den bisherigen rein passiven Schutzmöglichkeiten der Fall war. Der zu überwachende Automat wird dazu von dem vorgestellten Sensorsystem an sicherheitsrelevanten Oberflächen mittels piezolelektrischer Folien überwacht.

Keywords: Körperschallsensor, Parkscheinautomaten, PVDF-Folien, Structural Health Monitoring

\section{Problemstellung und Motivation}

In Deutschland und auch im Ausland sind Automaten im öffentlichen Raum verstärkt das Ziel von kriminellen Aktivitäten. Als Beispiel seien Parkscheinautomaten (PSA) angeführt, die aufgrund der BargeldAufbewahrungsfunktion immer wieder in den Fokus von organisierten Täterbanden und Einzelpersonen rücken [1], siehe Abb. 1. Bundesweit verzeichnete allein die Deutsche Bahn 330 Fälle von Automatensprengungen im Jahr 2012 und 480 Fälle im Jahr 2013. Weitere Angriffsmethoden auf Automaten sind zahlreiche Einbruchversuche mit Werkzeugen wie Schneidbrennern, Vorschlaghämmern, Metallsägen oder Brechstangen. Auch hierbei werden die Automaten teils irreparabel zerstört und können nur noch teilweise oder gar nicht mehr ihrer ursprünglichen Verwendung entsprechend genutzt werden, was neben den Reparatur- auch Ausfallkosten nach sich zieht. $\mathrm{Da}$ die entstehenden Sachschäden dabei häufig den Wert der Beute um ein Vielfaches übersteigen, entsteht den Automatenbetreibern allein schon durch das Eindringen in den Automaten ein erheblicher Schaden. Aber auch erfolglose Einbruchversuche, bei denen der oder die Täter den Automatentresor letztlich nicht öffnen können, verursachen für den Automatenbetreiber aufgrund der mechanischen Beschädigungen hohe Kosten bis hin zum wirtschaftlichen Totalschaden.

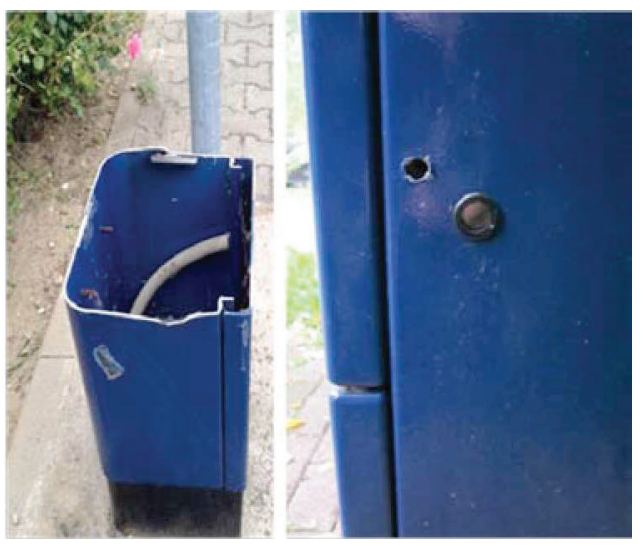

Abb. 1: Zerstörte Automaten nach Einbruchversuchen.

Der Fokus dieses Projektes liegt daher insbesondere auf der frühzeitigen Erkennung und Meldung von gewaltsamen Aufbruchversuchen und Vandalismus, um die Täter dadurch vor der weiteren oder sogar 
vollständigen Zerstörung des Automaten abzuhalten.

Sobald ein Angriff erkannt wird, können geeignete Gegenmaßnahmen eingeleitet werden. Das kann zum Beispiel ein lautstarker akustischer Alarm sein, durch den der oder die Täter sich ertappt oder gestört fühlen und in Folge von ihrem Vorhaben absehen. Dies ist insbesondere bei Einzeltätern und Vanadalismus zu erwarten. Im Bereich der Organisierten Kriminalität (OK) werden sich die Täter mutmaßlich nicht durch akustische Signale abschrecken lassen. Werden aber innerhalb eines kurzen Zeitraums viele Automatenaufbrüche erkannt, könnte so eine Aufbruchserie der OK zugeschrieben werden, was den Fahndungsdruck auf die Täter erhöht. Daher ist durch dieses System auch eine abschreckende Wirkung auf die Organisierte Kriminalität möglich. Des Weiteren könnte eine gezielte Zerstörung der vorhandenen Wertgegenstände erfolgen, was den Angriff zwecklos und weitere Angriffe in der Zukunft zunehmend unattraktiv machen würde.

Ein wesentlicher Vorteil des hier vorgeschlagenen Systems ist, dass die Angriffe auf den Automaten praktisch sofort und vollautomatisch erkannt werden können. Auf diese Weise soll ein effektiverer Schutz vor der vollständigen Zerstörung des Automaten gewährleistet werden.

\section{Stand der Technik}

Im Folgenden wird zunächst ein Überblick über die passiven Schutzsysteme von Automaten gegeben. Anschließend werden technische Systeme zur Verhinderung von Einbrüchen vorgestellt, bevor eine Übersicht über Körperschallsensoren und ihre heutigen Einsatzgebiete in der Industrie folgen. Abschließend werden verschiedene Messwertaufnehmer für Körperschall vorgestellt.

Die Widerstandsfähigkeit von Parkscheinautomaten gegen Einbruchdiebstahl wird mittels der VDSRichtlinie für mechanische Sicherungseinrichtungen klassifiziert [2]. Die Automaten werden dabei in die Sicherheitsklassen P1 bis P4 eingeteilt, wobei die Anforderungen hinsichtlich der Dauer bis zu einer möglichen Überwindung des mechanischen Schutzes und die Wirksamkeit der eingesetzten Werkzeuge von der niedrigsten Klasse P1 bis zur höchsten Klasse P4 zunehmen. Die Sicherheitsklassen von Parkautomaten beziehen sich dabei auf einen rein passiven Schutz vor Einbrüchen. So muss etwa ein Automat der Sicherheitsklasse P1 eine Widerstandszeit von 3 Minuten bezüglich der Grundwerkzeugsätze A und B erfüllen. Als mechanische Einbruchverhinderung können etwa stärkere Wandbleche, das Unterbinden von Angriffspunkten für Hebelwerkzeuge oder stabilere Türscharniere genannt werden.

Systeme zur Erkennung und Verhinderung von Einbruchversuchen an Automaten sind bereits heute im Einsatz. Dies geschieht zum Beispiel mit einer Überwachung durch Kameras im Automaten und in der Umgebung, Erschütterungs- und Körperschall-Sensoren, Positionierungssystemen (GPS o.Ä.), Bewegungsmeldern oder einer Fremdkörpererkennung in den Eingabefächern. Des Weiteren wurden Gas Protection Units entwickelt, deren GasSensoren erkennen ob ein Gas in den Automaten eintritt und dann bei Bedarf einen Alarm auslösen oder versuchen, das Gas zu neutralisieren.

Daneben existieren Systeme die Automaten schützen sollen, die nicht im öffentlichen Raum stehen und jederzeit zugänglich sein müssen. Solche Sensoren, die Einbruchversuche verhindern sollen, werden beispielsweise in Gebäuden oder Grundstücken eingesetzt, welche den Automaten umgeben bzw. inn schützen. Hier sind zum Beispiel Flächenlaser, Sensorkabel für Zaunüberwachung, Lichtschranken oder Brandmeldesysteme entwickelt worden, die es erlauben die Objekte zu überwachen bei denen kein Publikumsverkehr erlaubt ist. $\mathrm{Da}$ dies für Fahrkarten- und Geldautomaten in aller Regel nicht der Fall ist, werden diese Systeme selten für die Einbruchüberwachung in diesem Bereich eingesetzt.

Es werden bereits heute Körperschallmelder zum Schutz von vorzugsweise Großraumtresoren in Banken und Tresoren in Automaten auf dem Markt angeboten, beispielsweise [3]. Von verschiedenen kommerziellen Anbietern wurden Sensoren für die kontinuierliche Überwachung von Safes, Geldautomaten, Nachttresoren, Sicherheitsräumen und Stahlschränken entwickelt die einen Schutz vor sehr schweren Angriffen gewährleisten sollen. Die Systeme erkennen beispielsweise Angriffe durch Explosionen, Diamantbohrer, Schlagbohrer und Sauerstofflanzen. Hierfür werten die Körperschallmelder dann mit Hilfe einfacher Algorithmen Zeitverlauf, Frequenz und Amplitude aus. Die Sensoren verfügen häufig über Empfindlichkeitseinstellungen, die es dem Anwender erlauben, die Systeme in Umgebungen mit mehr oder weniger starken Störgeräuschen und auf unterschiedlichen Materialien wie Beton, Stahl oder auch synthetischen Materialien zu verwenden. Der 
Erfassungsbereich wird dabei mit bis zu $18 \mathrm{~m}$ auf Beton unter Verwendung mechanischer Werkzeuge angegeben und ist damit vorzugweise für die Überwachung von Großraumtresoren ausgelegt.

Das am Institut bearbeitete BMBFForschungsprojekt "KESS - Konfigurierbares elektronisches Schadenidentifikationssystem" beschäftigt sich mit der Erkennung von Bagatellschäden auf der Außenhaut von Automobilen. Hierfür ist ein intelligentes Körperschallsensornetzwerk entwickelt worden, das eine optimale Überwachung der Karosserieoberfläche gewährleistet [4]. Der bei der Entstehung des Schadens ausgesandte Körperschall wird mithilfe des Sensornetzwerks in Echtzeit aufgenommen und bewertet. Der Schadenort wird über eine spektrale Zeitdifferenzmessung bestimmt [5] und zusammen mit der ermittelten Schadenbewertung wird eine Prognose erstellt. Hierfür sind im Rahmen dieses Projektes spezielle Algorithmen und Sensoren entwickelt worden, die bereits Bestandteil mehrerer Patente sind [6] [7].

Körperschallüberwachung wird heutzutage auch bei der Überwachung des Zustands von Strukturen (engl. Structural Health Monitoring (SHM)) erfolgreich eingesetzt. Typische Einsatzgebiete sind hier zum Beispiel Brücken und Gebäude [8]. Die installierten Sensoren liefern Informationen, die Rückschlüsse auf den Materialzustand zulassen. Damit lässt sich eine Prognose erstellen, ob und zu welchem Zeitpunkt eine Wartung der beanspruchten Struktur notwendig ist [9]. Die unterschiedlichen Messverfahren können somit eine Möglichkeit darstellen, kostenintensive Wartungen so weit wie möglich zeitlich nach hinten zu verlagern, ohne dabei die Gefahr einer sicherheitsrelevanten oder anderweitigen Schädigung der Objekte, wie zum Beispiel einer Zerstörung durch Rissentwicklung [10], einzugehen.

Im Bereich der Zustandsüberwachung durch Körperschallanalyse gewinnt zunehmend auch das maschinelle Lernen an Bedeutung. Die automatische Klassifikation der Messsignale erfolgt dabei häufig auf Basis von Vektor- oder Folgenklassifikatoren. In [11] erfolgt die Modellierung der Signale mittels HiddenMarkov-Models (HMM). Für die Merkmalsextraktion wird die Fouriertransformation verwendet, mit anschließender Merkmalskompression. Die Wissensquellen des Klassifikators werden mit einer manuell klassifizierten Trainingsstichprobe (Supervised Learning) automatisch aufgebaut. Neben HMM werden auch Support Vector Machines (SVM) für die
Klassifikation akustischer Emissionen verwendet. In [12] wird eine Klassifizierung akustischer Emissionen mittels einer Multiclass SVM vorgestellt. Für die Transformation der Signale in den Analysebereich wird die Wavelet Packet Analysis verwendet. Für die Reduktion des Merkmalsraums werden sowohl Feature Extraction (FE) als auch Feature Selection (FS) verwendet. Die SVM wird dann mit der reduzierten Teilmenge des ursprünglichen Merkmalsraums trainiert. In [13] wird die Verwendung einer SVM für die Klassifizierung von akustischen Emissionen von Pipeline-Ventilen gezeigt. In [14] wird eine verallgemeinerte Theorie über die automatische Interpretation und die Anwendung maschinellen Lernens auf beliebigen Sensorsignalen vorgestellt.

Ein wichtiges Gebiet des Maschinellen Lernens ist die Merkmalsreduktion. Die Merkmalsreduktion kann dabei nach [15] prinzipiell in eine Merkmalsauswahl (Feature Selection) [16] und eine Merkmalsextraktion (Feature Extraction) unterteilt werden. Die Merkmalsauswahl reduziert den Merkmalsraum dadurch, dass nur solche Merkmale für die Klassifikation verwendet werden, die auch die Unterscheidbarkeit der Klassen erhöht. Bei der Merkmalsextrahierung werden neue Merkmale durch die Transformation oder Kombination einzelner Merkmale konstruiert.

\section{Systemübersicht}

Das in diesem Beitrag vorgestellte körperschallbasierte Sensorsystem gliedert sich gemäß Abb. 2 in die folgenden funktionalen Komponenten: Eine PVDF-Folie als Messwertaufnehmer (MWA), eine analoge Signalvorverarbeitung und eine digitale Signalverarbeitungs-Einheit, eine Spannungsversorgung für die Sensorelektronik sowie die externen Schnittstellen.

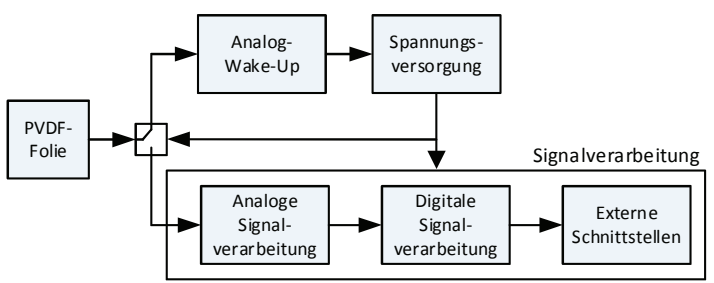

\section{Abb. 2: Funktionale Komponenten des Sensors und deren Signalpfade.}

Als Messwertaufnehmer werden an diesem Sensor bis zu sechs piezoelektrische PVDFFolien verwendet, die parallel abgetastet werden können. Das Ausgangssignal der Messwertaufnehmer wird im Ruhezustand von 
der Analog-Wake-Up Einheit (AWU) ausgewertet. Bei einem vermuteten Angriff schaltet der AWU die Spannungsversorgung für die Signalverarbeitung ein und die Signalleitung der MWA wird von der AWU auf die analoge Signalvorverarbeitung umgeschaltet.

Die Signale werden in der Signalvorverarbeitung zunächst impedanzgewandelt und dann bandpassgefiltert. Anschließend findet auf einem FPGA und einem digitalen Signalprozessor (DSP) die frei programmierbare digitale Signalverarbeitung statt. Die Sensorelektronik mit Messwertaufnehmer ist in Abb. 3 zu sehen.

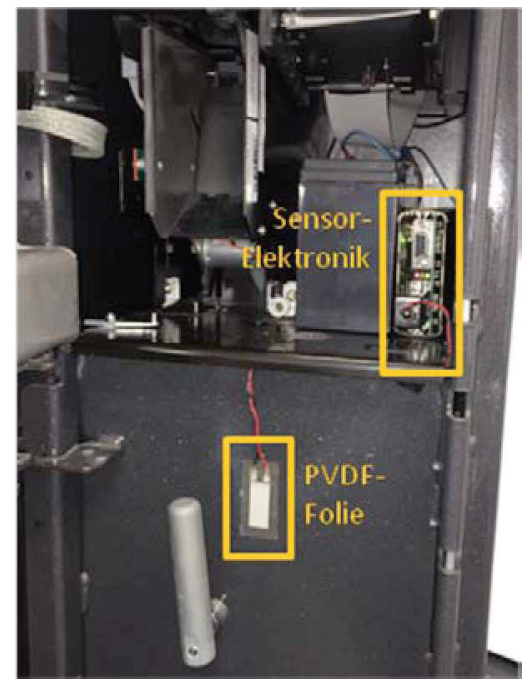

Abb. 3: Der vorgestellte Körperschallsensor verbaut in einem Automaten.

Über die externen Schnittstellen kann das System neben seiner sensorischen Tätigkeit auch zusätzlich oder ausschließlich als Messsystem zur Rohdatenaufnahme betrieben werden. Damit ist es möglich, die Datenbasis des lernenden Systems mit derselben Verarbeitungskette zu erstellen, mit der die Signale auch im operativen Einsatz analysiert werden. Für die Messwertaufnahme wird kein weiterer Signalverarbeitungspfad benötigt, da die Rohwerte direkt von der digitalen Signalverarbeitungs-Einheit auf einem nichtflüchtigen Speicher abgelegt werden.

Die Sensortopologie wurde auf Basis definierter Angriffsfälle ausgelegt. Dafür wurde auf den Werkzeugsatz A der Grundwerkzeugsatz der Prüfwerkzeuge in in der VDS-Richtlinie für mechanische Sicherungseinrichtungen zurückgegriffen [2].

Hieraus wurde ein technisches Konzept für den intelligenten Sensor entworfen. Dabei wurde festgelegt, wie viele Messwertaufnehmer (MWA) verwendet werden und auf welchen Oberflächen des Automaten diese anzubringen sind, damit der gesamte Automat bezüglich der Angriffserkennung optimal überwacht werden kann.

Tab. 1: Werkzeugsatz A der VdS-Richtlinien für mechanische Sicherungseinrichtungen.

\begin{tabular}{|c|c|}
\hline Bezeichnung & Spezifikation \\
\hline Schraubendreher & Klingenbreite \\
& $10 \mathrm{~mm}$ \\
\hline Schraubendreher & $\begin{array}{c}\text { Klingenbreite } \\
14 \mathrm{~mm}\end{array}$ \\
\hline Holzkeile & $200 / 80 / 40 \mathrm{~mm}$ \\
& L/B/H max. \\
\hline Kunstoffkeile & $200 / 80 / 40 \mathrm{~mm}$ \\
& L/B/H max. \\
\hline Wasserpumpenzange & Länge $240 \mathrm{~mm}$ \\
\hline Rohrzange & Länge $240 \mathrm{~mm}$ \\
\hline
\end{tabular}

\section{Messwertaufnehmer}

Als Messwertaufnehmer werden Polyvinylidenfluorid-Foliensensoren (PVDFFolien) eingesetzt. Diese MWA haben aus Sicht von Low-Power-Sensoren den Vorteil, dass für ihre sensorische Eigenschaft keine Energie verbraucht wird, da sie rein passiv betrieben werden. Die Ausgangsgröße dieser Messwertaufnehmer ist eine Spannung, die auf dem piezoelektrischen Effekt beruht.

Der Eingang dieser Elektronik ist für den Einsatz von bis zu sechs einzelnen Messwertaufnehmern ausgelegt und wird im Rahmen dieses Projekts mit einem dreikanaligen und drei weiteren je einkanaligen Messwertaunehmern betrieben. Der dreikanalige Messwertaunehmer ist in Abb. 4 gezeigt.

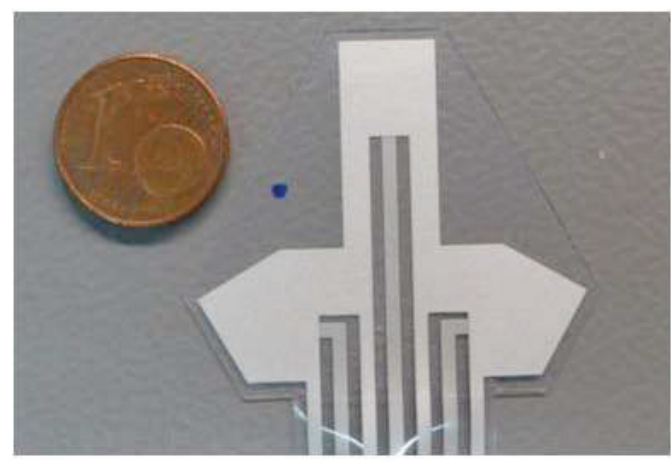

Abb. 4: Dreikanaliges Piezofilmelement mit einer Ein-Eurocent-Münze zum Größenvergleich.

Der dreikanalige MWA wurde am Institut entwickelt und kann zur Ortung von Schlagschäden verwendet werden [5]. Aufgrund des Designs ist eine sehr kompakte 
Sensor-Elektronik für die Ortung auf der Oberfläche des Automaten möglich. Die Ortung der Schallquelle kann weitere Hinweise über die Quelle und das Gefahrenpotenzial liefern. So wäre ein Tritt in der Nähe des Fundamentes ein Hinweis auf ein geringes Gefahrenpotenzial während ein Bohrgeräusch auf Höhe der Geldkassette einen akuten Angriffsfall nahe legt.

Aufgrund der bekannten Geometrie des dreikanaligen MWA kann, aus den Laufzeitdifferenzen des Körperschalls zwischen den sensorischen Kontaktflächen, die Einfallrichtung der Körperschallquelle zum Sensor rechnerisch ermittelt werden. Der Zusammenhang ist in Abb. 5 gezeigt.

Zudem weisen die Biegewellen in dem betrachteten Frequenzspektrum eine stark frequenzabhängige Ausbreitungsgeschwindigkeit auf. Für die BiegewellenAusbreitungsgeschwindigkeit gilt nach [17]:

$c_{\mathrm{B}} \approx \sqrt{1,8 \cdot h \cdot c_{\mathrm{L}} \cdot f}$

wobei $h$ die Dicke der Platte, $c_{\mathrm{L}}$ die Longitudinalgeschwindigkeit und $f$ die Frequenz ist. Die Wellenausbreitungsgeschwindigkeit nimmt also ungefähr proportional mit der Wurzel der Frequenz zu.

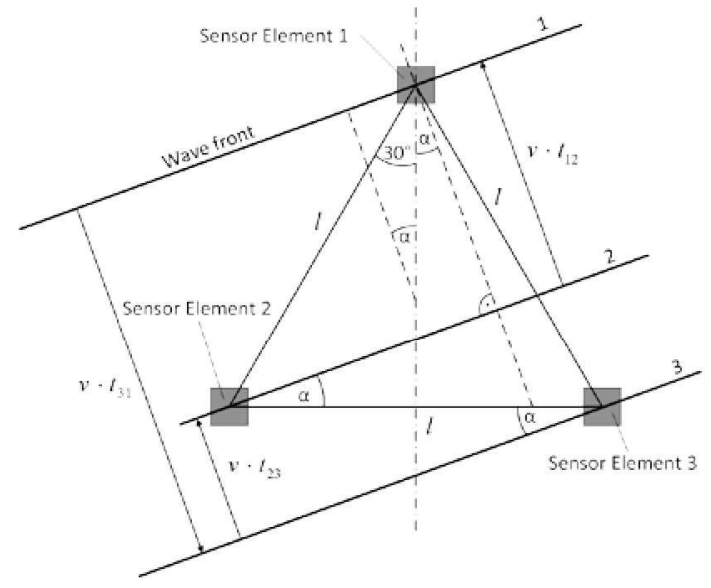

Abb. 5: Messwertaufnehmer mit ebener Wellenfront und geometrischen Beziehungen.

Für den Fall, dass der Körperschalleintrag der Automatenoberfläche impulsartig geschieht, kann die Bestimmung der Dispersion der Biegewelle genutzt werden, um den Abstand des Schlagschadens zum Sensor zu berechnen.

Die PVDF-Folien weisen zudem im unteren Spektralbereich eine Hochpass-Charakteristik auf. Da die Nutzsignale im Rahmen dieser Anwendung eher im oberen Frequenzbereich des hörbaren Körperschalls liegen, kommt diese Eigenschaft dem Projekt entgegen.

\section{Analog-Wake-Up}

Parkscheinautomaten werden häufig als netzunabhängige Inselsysteme betrieben. Da diese nicht an das Stromnetz angeschlossen sind, werden sie nur von einer Photovoltaikanlage mit angeschlossenem Akkumulator versorgt. Daher sollte ein Sensorsystem insbesondere im Kontext von Parkautomaten eine hohe Energieeffizienz gewährleisten.

Der hier vorgestellte Sensor befindet sich daher standardmäßig immer im Ruhezustand und die Signalverarbeitungs-Einheiten werden nicht mit Energie versorgt. Das heißt, es findet keine permanente Überwachung des Automaten durch die digitale Signalverarbeitung statt.

Der Umstand, dass die PVDF-Folien keine Energie für ihre Ausgangsspannung benötigen, wird daher in diesem Sensorsystem für eine weitere Funktion genutzt. Es wurde eine Analog-Wake-Up-Einheit entwickelt, deren Aufgabe es ist, die SignalverarbeitungsEinheiten möglichst nur dann einzuschalten, wenn eine potentielle Beschädigung des Automaten angenommen werden kann. Bei normalen Betriebs- und Störgeräuschen hingegen soll der Sensor im Ruhezustand bleiben, um den Energieverbrauch des Systems zu reduzieren. Der Sensor wird durch die Analog-Wake-Up-Einheit nur dann aufgeweckt, wenn die Ausgangsamplituden des MWA auf Kanal 0 einen definierten Schwellwert überschreiten und somit eine potentielle Beschädigung des Automaten angenommen werden kann.

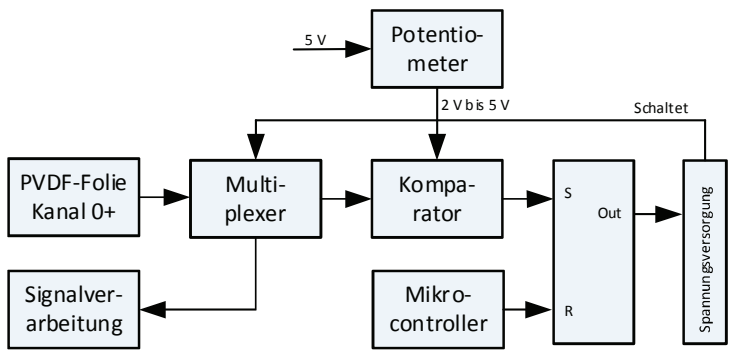

Abb. 6: Signalflussgraph des Analog-WakeUp.

Das Ausgangssignal des Messwertaufnehmers wird über einen Multiplexer im Ruhezustand des Sensors auf den Komparator geschaltet. Dieser vergleicht die Eingangsspannung mit dem über ein Potentiometer frei einstellbaren Schwellwert. Überschreitet die Amplitude des gefilterten Kanals den Schwellwert, wird ein Signal auf den Setz-Eingang des RS-Flipflop 
gegeben und somit die Spannungsversorgung des Signalverarbeitungspfads des Sensors eingeschaltet. Parallel wird über das Einschalten der Spannungsversorgung der Signalpfad des Messwertaufnehmers über den Multiplexer vom Komparator auf die Signalverarbeitung umgeschaltet. Hier werden die Signale dann weiter analysiert. Wenn innerhalb eines definierten Zeitraumes keine Beschädigung des Automaten erkannt wird, wird der Rücksetz-Eingang des Flipflops über den Mikrocontroller angesteuert. Somit wird die Spannungsversorgung wieder ausgeschaltet und der Signalpfad des Messwertaufnehmers wieder auf den Komparator geschaltet.

Die Leistungsaufnahme des Sensors liegt im Ruhezustand bei etwa $500 \mu \mathrm{W}$. Die analoge Signalvorverarbeitung hat eine Leistungsaufnahme von etwa $550 \mathrm{~mW}$. Die Sensorelektronik inklusive der digitalen Signalverarbeitungs-Einheit hat bei einer Taktung des DSP von $75 \mathrm{MHz}$ eine Leistungsaufnahme von etwa 1,44 W.

Die Zeit des Sensors bis zur vollständigen Betriebsbereitschaft beträgt etwa $40 \mathrm{~ms}$. Dies ist die Zeit, die der FPGA zum vollständigen Booten braucht. Da dieser die Daten des Analog-Digital-Umsetzers abholt, ist die Aufwachzeit des FPGA aktuell der begrenzende Faktor. Die Einschaltzeit der Spannungsversorgung des Sensors, nach Überschreiten des Schwellwerts, ist dem gegenüber sehr gering und nicht Gegenstand aktueller Optimierung. Es wird angenommen, dass ein erfolgreicher Angriff auf einen Automaten stets länger dauert, als das vollständige Aufwachen des Sensors.

Neben der Möglichkeit den Sensor im AnalogWake-Up-Modus zu betreiben gibt es auch die Option, den Sensor in einen Always-OnBetrieb umzuschalten. Hierfür wurde die Analog-Platine konfigurierbar entwickelt, um zwischen den beiden Betriebsmodi umschalten zu können. Das RS-Flipflop zum bedingten Einschalten der Elektronik kann überbrückt und der Sensor damit permanent eingeschaltet werden. Bei der Erstellung der Datenbasis des lernenden Systems ist einerseits eine Repräsentation der Signale nach dem Aufwachen des Sensors von Interesse, andererseits aber auch Daueraufnahmen zur Abschätzung der Stör- und Betriebsgeräusche. Der Always-On-Betriebsmodus ist dann notwendig, wenn die Elektronik als Messsystem betrieben werden soll, um kontinuierliche Aufnahmen von Körperschallgeräuschen und -szenarien zu erstellen. Diese Funktionalität ist vor allem in der Entwicklungs- und Erprobungszeit von Interesse und könnte, ebenso wie die externen
Schnittstellen zur Datenspeicherung, in einem reinen Produktivsystem weggelassen werden.

\section{Spannungsversorgung}

Die Elektronik wird im Rahmen dieses Projektes mit einem $12 \mathrm{~V}$ Akkumulator versorgt. Die Spannungsversorgung ist mit einer Suppressordiode vor Überspannungen und kurzzeitigen Spannungsimpulsen geschützt. Über einen Abwärtswandler mit einem Eingangsspannungsbereich von $6 \mathrm{~V}$ bis $18 \mathrm{~V}$ wird eine $5 \mathrm{~V}$ Spannung bereitgestellt, die die AWU versorgt.

Die AWU schaltet im Angriffsfall über einen High-Side-Schalter die Spannungsversorgung für den Signalverarbeitungspfad ein. Über lineare Längsregler werden die verschiedenen Spannungsebenen für die Signalverarbeitungskomponenten zur Verfügung gestellt. Hierfür werden Low-DropSpannungsregler verwendet. Wobei die $5 \mathrm{~V}$ Spannung des Abwärtswandlers auch zur Versorgung des ADC verwendet wird. Diese Spannung wird über ein Glättungsfilter zur Verfügung gestellt, um die Welligkeit der Spannung weiter zur verringern. Das Blockschaltbild der Spannungsversorgung ist in Abb. 7 gezeigt.

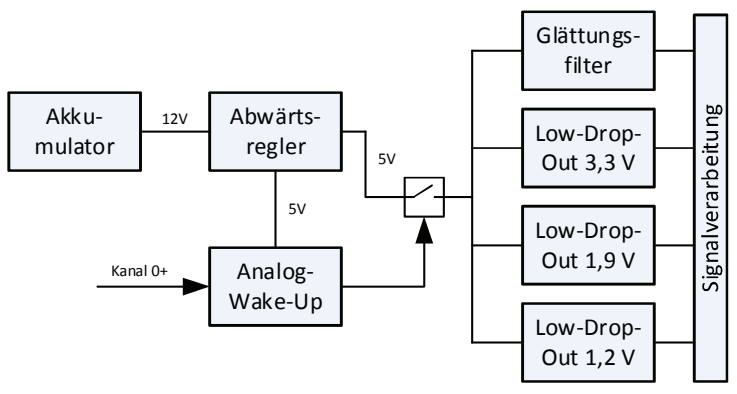

Abb. 7: Aufbau der Spannungsversorgung der Sensorelektronik.

\section{Analoge Signalvorverarbeitung}

Nach dem Einschalten des Signalverarbeitungspfads wird das MWASignal über den Multiplexer vom Komparator auf die analoge Signalverarbeitung umgeschaltet.

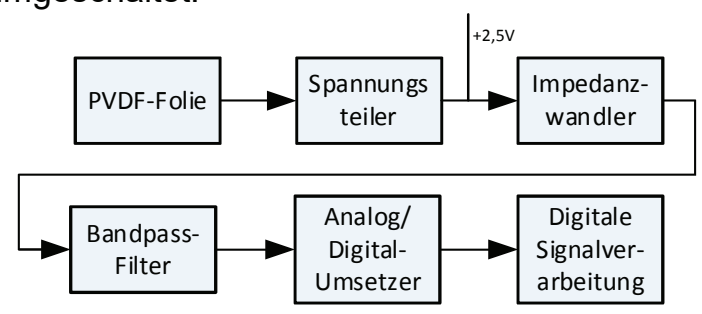

Abb. 8: Signalflussgraph der analogen Signalvorverarbeitung. 
Zunächst wird über einen Spannungsteiler das Signal der Messwertaufnehmer auf den Eingangsspannungsbereich des Analog/Digital-Umsetzers skaliert. Der ADC hat einen Eingangsbereich von $\pm 6 \mathrm{~V}$ und die PVDF-Folie ist mit einer Ausgangsspannung von $\pm 10 \mathrm{~V}$ angegeben. Die Ausgangssignalspannung wird daher über einen Spannungsteiler im Verhältnis 1,2 zu 5,9 geteilt und anschließend um 2,5 V hochgesetzt. Um die Signalspannung des hochohmigen Spannungsteilers nicht mit dem Bandpass zu belasten, wird sie mittels eines Spannungsfolgers entkoppelt. Hier kommt ein Operationsverstärker als aktiver Impedanzwandler zum Einsatz. Das gewandelte Signal wird von einem passiven Bandpass erster Ordnung gefiltert, um ein Aliasing im Frequenzbereich zu verhindern. Die untere Grenzfrequenz des Filters liegt bei etwa $30 \mathrm{~Hz}$, die obere Grenzfrequenz bei etwa $5 \mathrm{kHz}$. Hier wird auch der Gleichanteil des hochgesetzten Signals wieder herausgefiltert. Das Übertragungsverhalten des Bandpasses ist in Abb. 9 gezeigt.

Die gefilterten Signale werden anschließend von einem mehrkanaligen Analog/DigitalUmsetzer zeitsynchron und differentiell abgetastet. Die Abtastrate ist dabei für jeden Kanal auf den Audiofrequenzbereich beschränkt und liegt bei $11 \mathrm{kHz}$. Die Auflösung des ADC bei dieser Abtastrate beträgt 16 Bit.

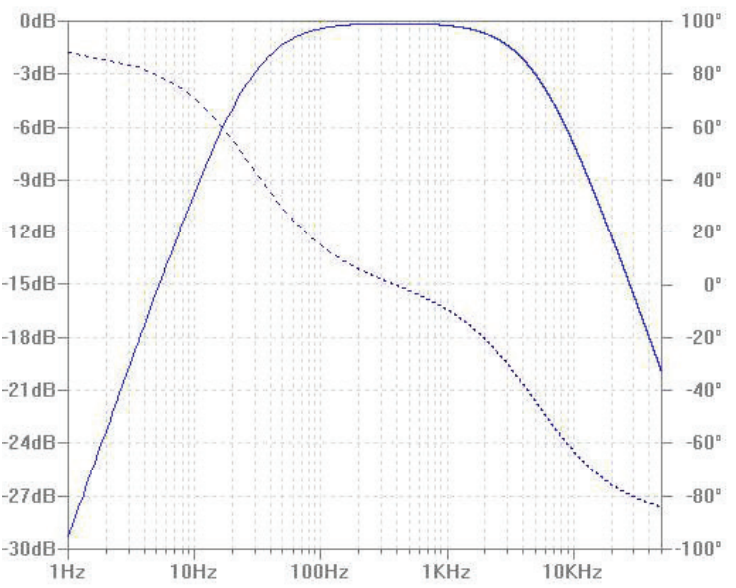

Abb. 9: Übertragungsverhalten des passiven Bandpasses; gestrichelte Linie: Phase in Grad; durchgezogene Linie: Amplitude in $d B$.

\section{Digitale Signalverarbeitung}

Die digitale Signalverarbeitungs-Einheit besteht aus einem Field Programmable Gate Array (FPGA) mit Anbindung an einen Digitalen Signalprozessor (DSP), sowie externen Schnittstellen zu einem SD-
Kartenspeicher und einem konfigurierbaren USB-Anschluss.

Das FPGA holt die digitalisierten Signale des A/D-Umsetzers über einen SPI-Bus ab und hält diese in einem internen Speicher bis zur weiteren Verarbeitung vor. Der Takt des FPGA wird dabei vom DSP vorgegeben. Es ist geplant die Fouriertransformation der Signale direkt auf dem FPGA durchzuführen, da Signalverarbeitung fensterweise und die Berechnung mittels einer Implementierung der FFT erfolgen soll. Die vorverarbeiteten Daten werden dann über einen parallelen 16-Bit Bus an den DSP weitergegeben. Auf diesem wird die eigentliche Mustererkennung ausgeführt.

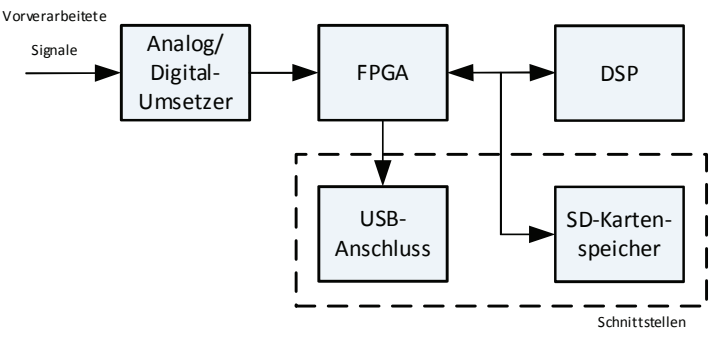

Abb. 10: Aufbau der Spannungsversorgung der Sensorelektronik.

Neben weiteren Schnittstellen, wurden zwei Schnittstellen speziell für die Speicherung von Messwerten zur Verfügung gestellt. Dabei handelt es sich zum Einen um einen USBAnschluss und zum Anderen um einen SDKartenspeicher. Der SD-Kartenspeicher kann hier sowohl über das FPGA als auch über den DSP beschrieben werden. Der USB-Port wird über einen eigenen Bus direkt vom FPGA angesprochen.

\section{Verwendung als Messsystem}

Bei der Entwicklung des Sensors wurde berücksichtigt, dass dieser auch die Rohwerte der Messwertaufnehmer sowie die internen Zustände der Signalverarbeitung speichern können soll. Aus diesem Grund ist ein SDKartenspeicher verbaut worden, auf dem die Rohdaten des A/D-Umsetzers direkt vom FPGA, als auch die verarbeiteten Daten der digitalen Signalverarbeitung vom DSP gespeichert werden können. Ebenfalls ist ein USB-Anschluss verbaut worden, über den die Daten an einen externen Rechner gesendet werden können. Durch das Speichern der Rohdaten kann die Aufnahme der Daten über denselben Signalpfad erfolgen, wie die spätere Signalverarbeitung im Produktiveinsatz. Die externen Schnittstellen sind insbesondere in der Entwicklungsphase von Bedeutung und können später entfallen. 


\section{Ausblick}

In diesem Beitrag wurde das entwickelte und aufgebaute körperschallbasierte Sensorsystem vorgestellt. Im nächsten Schritt ist geplant mit dem Sensorsystem Messaufnahmen in Parkscheinautomaten durchzuführen. Dabei sollen sowohl die Umgebungs- und Störgeräusche im Produktivbetrieb der Automaten, als auch die Bedien- und Nutzungsgeräusche aufgenommen werden. Im Anschluss werden die Aufbruchversuche an einem Test-Automaten mit den definierten Prüfwerkzeugen nachgestellt. Auf Basis dieser Messdaten soll dann eine Mustererkennung entwickelt werden, die mit hoher Treffsicherheit die Angriffe auf Automaten erkennen kann. Diese Mustererkennung soll anschließend auf den Sensor portiert werden. Perspektivisch soll die Möglichkeit zur Nutzung des Sensors als Service-Einheit zur Prädiktion des Abnutzungsvorrats von Verschleißteilen geprüft werden.

\section{Literaturnachweis}

[1] Umsetzung der VdS-Prüfrichtlinie 3546 für mechanische Sicherungseinrichtungen Parkscheinautomaten - Anforderungen, Klassifizierung und Prüfmethoden.

[2] VdS-Richtlinien für mechanische Sicherungseinrichtungen - VdS 3546

[3] Siemens, GM730 Körperschallmelder - Senstec Körperschallmelder

[4] S. Gontscharov, K.-L. Krieger, et al.: Verteilte Intelligenz in einem Sensornetzwerk zur Körperschalldetektion in Fahrzeugen, Embedded Systems Symposium, München, 2013.

[5] J. Harmstorf, A. Kneifel, K-L. Krieger, Lokalisierung von Schlagschäden auf dünnwandigen Strukturen. Deutsche Jahrestagung für Akustik, Nürnberg, 2015.

[6] DE 102013016165.7 „Kriterien zur Bewertung von Körperschallsignalen zur Erkennung von Beschädigungen".

[7] DE 102012023 393.A1 „Verfahren zur Aufnahme fahrzeugrelevanter Daten, insbesondere zur Erfassung und zur Bewertung von Bagatellschäden, Sensoranordnung zum Einbau in ein Fahrzeug und Fahrzeug mit der Sensoranordnung zur Durchführung des Verfahrens"

[8]C. Liu, J.T. DeWolf, J.-H. Kim, Development of a baseline for structural health monitoring for a curved post-tensioned concrete box-girder bridge, Engineering Structures, Vol. 31, Issue 12, 3107-3115, 2009.

[9] S. Kim, et al., Health Monitoring of Civil Infrastructures Using Wireless Sensor Networks, IEEE, Information Processing in Sensor Networks, 2007.

[10] Q. Wang, J. Xu, Lamb wave tomography technique for crack damage detection, IEEE, Proceedings of the 33rd Chinese Control Conference, 2014.
[11] C. Tschöpe, M. Wolff, Statistical Classifiers for Structural Health Monitoring, IEEE Sensors Journal, Vol. 9, 2009.

[12] J. Zhao, K. Wang, Y. Guo, Acoustic Emission Signals Classification Based on Support Vector Machine, IEEE Computer Engineering and Technology (ICCET), 2010.

[13] Z. Haifeng, L. Zhenlin, J. Zhongli, et.al., Application of acoustic emission and support vector machine to detect the leakage of pipeline valve, Fifth Conference on Measuring Technology and Mechatronics Automation, 2013.

[14] M. Wolff, C. Tschöpe, Pattern Recognition for Sensor Signals, IEEE Sensors Conference, 2009.

[15] P. Somol, J. Novovicova, P. Pudil, Efficient Feature Subset Selection and Subset Size Optimization, Pattern Recognition, Recent Advances, Adam Herout (Ed.), 75-95, 2010.

[16] A. Salappa, M. Doumpos and C. Zopounidis, Feature selection algorithms in classification problems: an experimental evaluation, Optimization Methods and Software, Vol. 22, No. 1, 199-214, 2007.

[17] L. Cremer, M. Heckl, B.A.T. Petersson: Structure-Borne Sound: Structural Vibrations and Sound Radiation at Audio Frequencies. Springer-Verlag, Berlin Heidelberg New York, 3rd edition (2005)]: 